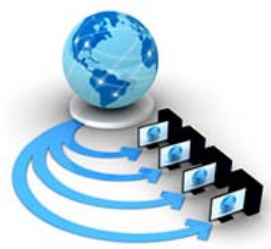

Volume 9, No. 3, May-June 2018

RESEARCH PAPER

\title{
COMPARISON OF MULTIMODAL TUMOR IMAGE SEGMENTATION TECHNIQUES
}

\author{
D. Moitra \\ University of North Bengal, \\ India
}

\begin{abstract}
Use of multimodal imaging for the classification of tumors in human body is on the rise. Segmentation is an important step of such classification process. There is need of carrying out a benchmark study by considering the leading segmentation techniques. This may help researchers in future to select a better segmentation technique.
\end{abstract}

Keywords: Multimodal, Tumor, Segmentation, Benchmark

\section{Introduction}

Of late, multimodal bio-medical imaging techniques are gaining rapid popularity [5]. In case of detecting a tumor in human body, such techniques are very useful, too. The reason behind this is manifold. First of all, the advantages of both structural and functional imaging techniques will be there together. They will also cancel out the disadvantages of each other. For example, hyper metabolism may look like a tumor in a typical PET image, but such problem may be avoided by using PET/CT or MRI-PET. Such resemblance can hardly deceive MRI as it relies on the anatomical nature of the tumor. On the other hand, a leakage at the boundary region of a tumor may create problem in case of MRI images, but such problem may be handled more efficiently by using MRI-PET. The ability of PET to identify any functional change (chemical or metabolic) in human body will be very handy in such cases. Resolution or noise related problems may also be overcome to a greater extent by using multimodal imaging techniques. Thus, multimodal medical imaging techniques may contribute more significantly in diagnosing even very small tumor in human body, than any individual functional or structural imaging technique.

\section{Segmentation}

Segmentation of tumor images has been a pivotal technique to group similar pixels and to properly identify the Region of Interest [4]. So far, many segmentation techniques have been proposed [1]. They may be classified into categories like thresholding based, texture based, deformable model based, stochastic, learning based, etc. [6]. Gradient based or spectral based categories may also be considered as important segmentation techniques. Proper segmentation helps in classifying tumors in a far more accurate way. In turn, it also helps radiologists or oncologists to formulate an efficient way of treatment.

\section{Objective}

In the case of automated classification of tumors, segmentation is an essential and a very common step. Selection method of such segmentation technique has been often found arbitrary in contemporary research papers [3]. A few researchers have tried to compare different segmentation techniques individually for functional or structural bio-medical imaging [2]. In the case of multimodal imaging such efforts are quite infrequently visible. There is a need to carry out a study to evaluate various major segmentation techniques with respect to different kinds of tumors commonly found in human body. The objective of the present study is to benchmark the performance of prime segmentation techniques while being applied on multimodal tumor images and that too, of tumors found in different parts of human body. The rest of the study is comprised of the following: section 4 is about the methodology, section 5 depicts result \& analysis, section 6 contains the conclusion and section 7 is regarding the future scope. To the best of our knowledge, such a vast comparative study on segmentation techniques has not been conducted so far.

\section{Methodology}

Six types of tumors, namely, Head \& Neck, Lung, Renal, Prostate, Thyroid and Breast tumors were considered for the study. Data of 120 patients was extracted from The Cancer Imaging Archive (TCIA). On an average, 20 DICOM images for each of the aforementioned categories of tumors were used in the study. The modalities of all the images were PET/CT. The following segmentation techniques were implemented: Region Growing (region based), Active Contour (deformable model based), Watershed (gradient based), Texture based (morphological), and Fuzzy Clustering Means (learning based). The accuracy of the 
segmentation result was measured by the Dice Similarity Coefficient (DSC):

$$
\operatorname{DSC}(\mathrm{V} 1, \mathrm{~V} 2)=2 *(|\mathrm{~V} 1 \& \mathrm{~V} 2|) /(|\mathrm{V} 1|+|\mathrm{V} 2|)
$$

Where V1 is the segmented volume and V2 is the surrogate truth. DSC is the ratio of sensitivity (true positive rate) and specificity (true negative rate). Each segmentation algorithm was executed against all the data from each category of tumors and the average DSC value was recorded subsequently. All the algorithms were implemented in Matlab R2015a.

\section{Result \& Analysis}

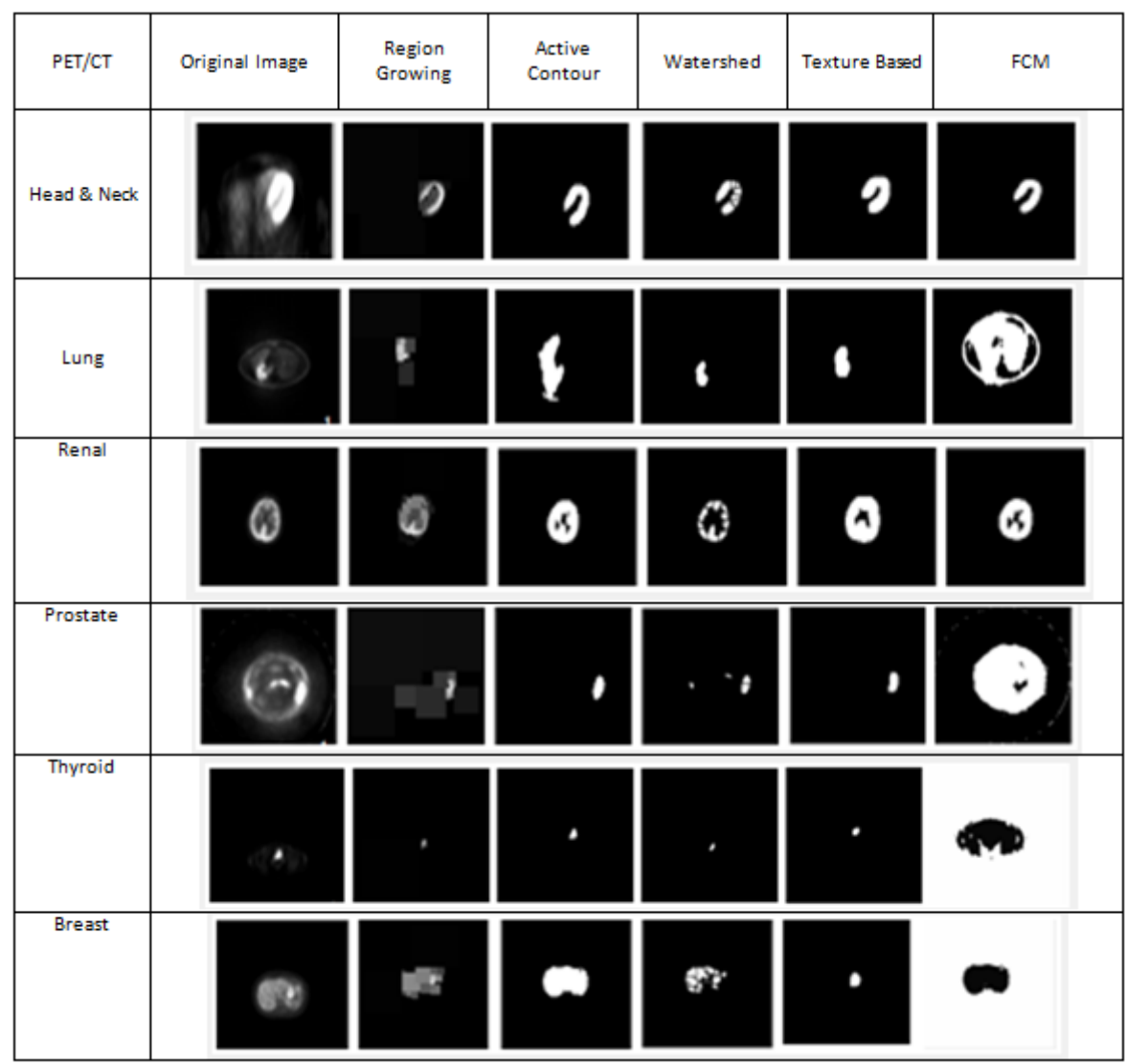

Figure1: Sample result of the comparison of segmentation techniques

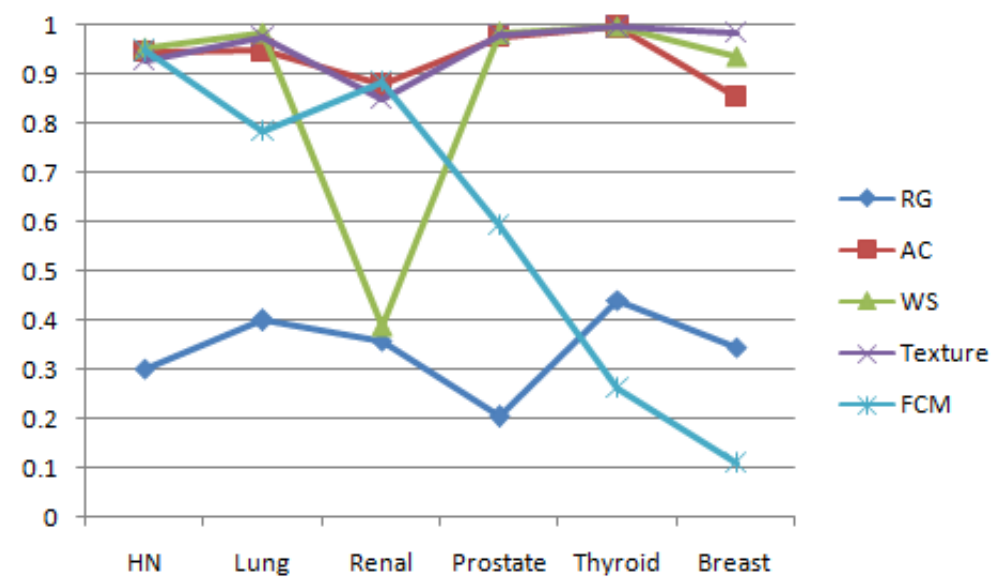

Figure2: Comparison of DSC values 
For Head \& Neck tumors almost all the segmentation techniques except the Region Growing method did a pretty good job. For Renal tumors - Active Contour, Texture and FCM methods had close calls. For Prostate, Thyroid and Lungs tumors - Texture, Watershed and Active Contour methods had a neck and neck situation. For Breast Tumors, the results of Watershed and Texture techniques were quite commendable.

\section{Conclusion}

From Figure1 and Figure2, it was evident that the texture based segmentation technique had been a consistent performer for almost every type of tumors under consideration. It was also found that, Active Contour and Watershed segmentation techniques might also be useful in some cases. The performances of other techniques were not very consistent. Although in some cases a few techniques did well, e.g. FCM was effective while segmenting Head \& Neck or Renal tumors. In fine, we may conclude that, the texture genre of segmentation is quite handy as far as the automatic segmentation of tumors in human body is concerned.

\section{Future Scope}

In future, such a study may be conducted with more varieties of tumors and segmentation methods. Other prominent multimodal imaging techniques such as MRI-PET may also be used for conducting such a study. The outcome of texture (morphological) based segmentation may further be explored while classifying tumors in human body.

\section{Conflict of Interest}

None

\section{Ethical approval}

For this type of study formal consent is not required.

\section{Informed consent}

Not applicable.

\section{References}

[1] Foster B, Bagci U, Mansoor A, Xu Z, Mollura DJ. A review on segmentation of positron emission tomography images. Comput Biol Med.;50:7696(2014)

[2] Zaidi H, Abdoli M, Fuentes C, El Naqa I. Comparative methods for PET image segmentation in pharyngolaryngeal squamous cell carcinoma. Eur J Nucl Med Mol Imaging 39:881-91.10.1007/s00259011-2053-0(2012)

[3] Abir Baâzaouia,Walid Barhoumia, Ezzeddine Zagroubaa and Rostom Mabroukb, A Survey of PET Image Segmentation: Applications in Oncology, Cardiology and Neurology, Current Medical Imaging Reviews, Vol. 12, No. 1(2016)

[4] Moitra, Dipanjan. (2017). SEGMENTATION STRATEGY OF PET BRAIN TUMOR IMAGE. Indian Journal of Computer Science and Engineering 0976-5166. 8. 575-577

[5] Moitra, Dipanjan. (2017). Review of Brain Tumor Detection using Pattern Recognition Techniques. INTERNATIONAL JOURNAL OF COMPUTER SCIENCES AND ENGINEERING, Volume-5, Issue-2, E-ISSN: 2347-2693

[6] Brent Foster, Ulas Bagci, Awais Mansoor, Ziyue Xu, and Daniel J. Mollura, “A review on segmentation of positron emission tomography images”, Computers in Biology and Medicine, Vol. 50, pp.76-96, 2014. 